\title{
Risk factors for low bone mineral density and the 6-year rate of bone loss among premenopausal and perimenopausal women
}

\author{
Kathleen E. Bainbridge $\cdot$ MaryFran Sowers \\ Xihong Lin · Sioban D. Harlow
}

Received: 16 April 2003 / Accepted: 29 October 2003/Published online: 22 January 2004

(C) International Osteoporosis Foundation and National Osteoporosis Foundation 2004

\begin{abstract}
Risk factors that are associated with lower bone mineral density (BMD) may not necessarily be associated with increased bone loss among premenopausal and perimenopausal women. We determined risk factors for lower premenopausal and perimenopausal BMD while simultaneously determining risk factors for increased 6-year rate of bone loss among women aged 24-50 years within a population-based prospective cohort study. BMD of the lumbar spine and femoral neck, reported as $\mathrm{t}$ scores, were measured five times within the 6 -year study among 614 women who were between the ages of 24 and 44 in 1992/1993. Rates of bone loss were calculated from the repeated BMD measurements. Risk factors for lower BMD over time at the lumbar spine included history of any fracture $(P=0.005)$. The major risk factor for lower BMD over time at the femoral neck was family history of osteoporosis $(P<0.002)$. The major protective factor for greater BMD over time at both skeletal sites was additional body weight $(P<0.0001)$. Other protective factors for greater BMD over time at the femoral neck were modest alcohol consumption $(P=0.0002)$ and high-school sports participation $(P=0.002)$. Risk factors for greater bone loss
\end{abstract}

K.E. Bainbridge $\cdot$ M. Sowers $(\varangle) \cdot$ S.D. Harlow

Department of Epidemiology,

School of Public Health,

University of Michigan,

Ann Arbor, Michigan, USA

M. Sowers

Suite 310, 339 East Liberty,

Ann Arbor, Michigan 48104, USA

E-mail: mfsowers@umich.edu

Tel.: + 1-734-9363892

Fax: + 1-734-9988027

X. Lin

Department of Biostatistics,

School of Public Health,

University of Michigan,

Ann Arbor, Michigan, USA at either skeletal site included postmenopausal status ( $P<0.0001$ at the lumbar spine; $P=0.01$ at the femoral neck), and the reporting of a reproductive cancer $(P<0.0001$ at the lumbar spine; $P=0.0008$ at the femoral neck). Body weight was protective against bone loss at both skeletal sites $(P<0.0001)$. Baseline age, calcium intake, smoking, and current physical activity were not associated with BMD or bone loss. The understanding of the relative importance of risk factors for both low BMD and bone loss may assist in the identification of women at greater risk for subsequent low postmenopausal BMD.

Keywords Bone density - Bone loss - Longitudinal studies $\cdot$ Premenopause $\cdot$ Risk factors

\section{Introduction}

Low bone mineral density (BMD) in premenopausal or perimenopausal women can arise from their failure to accrue adequate peak bone mass, from loss of BMD subsequent to peak bone mass attainment, or both [1]. Although risk factors for low postmenopausal BMD have been well characterized, risk factors for low premenopausal BMD and for greater premenopausal and perimenopausal bone loss are not well understood, even though there is increasing evidence to suggest that bone loss is a process that begins prior to menopause [2, 3, 4]. The distinguishing of risk factors for low premenopausal BMD from those for premenopausal bone loss may assist in the identification of women at greater risk for low postmenopausal BMD.

Anthropometric, reproductive, and lifestyle-related exposures may contribute to the variation in either $\mathrm{BMD}$ or rate of BMD loss. Among premenopausal women, body weight has been consistently and positively associated with BMD [5, 6], although whether this factor is also related to bone loss is less clear. Irregular menses and amenorrhea have been consistently associated with lower BMD [7], but age at menarche has 
shown no consistent association with BMD or long-term bone change [8]. Most studies of the long-term effects of parity and lactation have found no association with BMD or bone loss, although several studies have observed considerable short-term bone loss during pregnancy and lactation that is recovered with the resumption of menses [9].

Dietary intake of calcium, vitamin D, dairy products, protein, and sodium, as well as supplemental calcium, have been evaluated for association with peak BMD or its loss, with no consensus $[10,11]$. Few studies of other nutrients, such as vitamin C $[12,13]$ and vitamin A [14], have been conducted, and they provide insufficient evidence regarding the role of such nutrients in BMD accrual or loss. Studies of other lifestyle factors such as alcohol consumption [15], tobacco smoking [16] and physical activity [17] in general populations are limited.

Lower BMD is associated with chronic illnesses such as asthma [18] and cancer [19], but the impact of these associations is not well documented in population-based studies. Because BMD is, in part, genetically determined $[20,21]$, an understanding of how family history might determine lower BMD and/or bone loss would facilitate the timing of intervention strategies. While lower BMD is the single best predictor of fracture among perimenopausal women [22], other data indicate that a history of fracture might indicate lower BMD [23, 24].

We undertook a large, population-based, prospective cohort study to evaluate the impact of selected anthropometric, reproductive, lifestyle, and medical history variables on BMD and bone loss, measured at the lumbar spine and the femoral neck, in women within an age range of 24 to 50 years. The specific aims were to (1) estimate measures of association between anthropometric, reproductive, dietary, lifestyle, and medical history variables and BMD, and (2) evaluate which of these variables is associated with a 6 -year rate of bone loss.

\section{Materials and methods}

This study is a component of the longitudinal Michigan Bone Health Study (MBHS) located in Tecumseh, Michigan, USA, a study that has been previously described [2]. Women were recruited for this population-based work from two sampling lists. The first list identified the female offspring of participants from the Tecumseh Community Health Study (TCHS), a population-based prospective cohort study established in 1959. In order to reflect the current community composition better, we used a second list to identify and facilitate recruitment of those women who were current community residents but whose families had not lived in Tecumseh when the TCHS began.

Women who were between the ages of 24 and 44 years in 1992 were eligible for inclusion in the MBHS. There were 674 female offspring identified from the first list who were still living in the community at study inception and who met the age criterion. Of these women, 539 (80\% participation) were successfully recruited through the use of letters, telephone calls and personal visits. An additional 135 female Tecumseh residents who met the age criterion were identified from Kohl's Directory, a list that included age, name, address and telephone number. Of these women, $121(87 \%$ participation) were enrolled. The total cohort, identified from the combined lists, included 660 eligible Caucasian women.
Reasons for non-participation included refusal $(<14 \%$ in any given year), having moved from the area (1-5\% in any given year), reporting significant health impairments (1-2\% in any given year), and six deaths. An annual BMD measurement was deferred to the following year if the woman was pregnant $(1-3 \%$ in any given year). More than $64 \%$ of the study population had bone measurements at all points in time, and almost $80 \%$ of the population had at least four of the five measures. Written informed consent was obtained annually from all participants, and approval for the conduct of the study was obtained from the University of Michigan Institutional Review Board.

\section{Bone mineral density}

At each data collection visit, BMD (in grams per square centimeter) was measured at the lumbar spine $\left(\mathrm{L}_{2}-4\right)$ and the femoral neck with dual-energy X-ray absorptiometry (DXA, DPX-L; Lunar Corp., Madison, Wis, USA). The same software (analysis software version 1.3y) was used for all data acquisition. The women were scanned by one of two certified technicians. Coefficients of variation for the DXA measurements determined from repeated measurements of phantoms were less than $1.0 \%$. We standardized BMD measurements relative to an unchanging database of normally distributed BMD measurements from a young, adult (aged 20-45), female population [25] to obtain t scores.

\section{Anthropometry}

At each examination the participants underwent a physical examination to provide the anthropometric measurements. Height (in centimeters) and weight (in kilograms) were measured with a stadiometer and a calibrated balance-beam scale, respectively, while participants wore a single layer of light clothing and no shoes. Body mass index (BMI) was calculated as weight in kilograms divided by the square of the height in meters and the women were categorized as underweight, normal, overweight, obese, and severely obese, with values of $20,25,30$, and $40 \mathrm{~kg} / \mathrm{m}^{2}$ as cutpoints [26]. Anthropometric measures were modeled as time-varying covariates in all repeated measures regressions.

\section{Reproductive history}

Assessment of reproductive history included self-reported age at menarche, number of pregnancies, history of breastfeeding, and menopausal status. Age at menarche was categorized to evaluate the extremes of the distribution relative to a referent group whose menarche was between 11 and 14 years, inclusive. The number of pregnancies was calculated as the sum of all a woman's pregnancies of at least 6 months' duration and categorized as $0,1,2,3$, and 4 or greater. Women who had had at least one pregnancy reported whether they had ever breastfed. Number of pregnancies and history of ever breastfeeding were modeled as summary measures with one value to represent the entire 6-year period.

Menstrual status was defined, based on each woman's self-report of the frequency of menstrual bleeds per year, self-report of gynecological surgery, and treatment with chemotherapy. Medical records were reviewed for $70 \%$ of the surgical cases, and, of these, confirmation of self-report occurred for $73 \%$ of single oophorectomies, $78 \%$ of hysterectomies, and $88 \%$ of double oophorectomies. Women were classified as premenopausal if they reported at least nine menstrual bleeds annually. Also classified as premenopausal were women reporting fewer than nine annual menstrual bleeds that could be explained by pregnancy, breast-feeding, single oophorectomy, use of a hormonal preparation that suppresses bleeding, or the use of fertility drugs with resumption of regular menses. Women who had undergone hysterectomies were considered as premenopausal if they had retained at least one ovary $(n=9)$. Otherwise, women reporting fewer than nine menstrual 
bleeds per year were classified as having irregular menses. Women were classified as postmenopausal if they reported the absence of a menstrual bleed for a 12-month period or a history of bilateral oophorectomy. Menstrual status was modeled as a time-varying covariate in repeated measures regression models.

\section{Dietary information}

At each of the four consecutive interviews between 1992 and 1993 and 1995 and 1996, dietary information was collected by use of the BlockNCI Health, History, and Habits Questionnaire [27]. The usual quantities of calcium (milligrams), vitamin A (international units), vitamin C (milligrams), sodium (milligrams), protein (grams) and total energy (kilocalories) intake per day were calculated via DIETSYS [28]. Energy values were calculated after we had excluded the contribution from alcohol consumption. Dietary vitamin D (IU) intake per day was calculated from reported frequencies of fortified cereal, milk, liver, liverwurst, and fish consumption, assuming a medium serving size and from values of vitamin $D$ content from a nutrient reference [29]. Depending on the year, data from between 55 and 73 cases were excluded from the dietary analysis because too few foods or too few calories were reported according to data checking standards. Nutrients and energy were modeled as time-varying covariates in the repeated measures regressions.

\section{Lifestyle}

At each interview, current physical activity level was measured by a self-administered Stanford five-city instrument [30] based on participant recall of minutes of physical activity during the previous January and the previous July. Intensity of physical activity was expressed in METS per week with one MET defined as the energy consumed per minute of sitting at rest. Current physical activity was modeled as a time-varying covariate in repeated measures regressions. During the 1993/1994 interview we ascertained past physical activity by asking the women if they had participated in high-school sports. Past physical activity was modeled as a summary measure.

At each interview, the women reported if they were current smokers, ex-smokers, or had never been a smoker. Lifetime smoking history was assessed upon the woman's entry into the study and updated when a woman reported a change in smoking status. Lifetime smoking history was modeled as a summary variable of a woman's smoking experience through 1998/1999. At each interview, the women reported if they had consumed any alcoholic beverages in the past year. Alcohol consumption was modeled as a time-varying covariate in repeated measures regressions.

\section{Medical history}

Medical history included a self-reported history of a breast, ovarian, or uterine cancer (combined as a single reproductive cancer variable) and modeled as a summary variable (any report/no report). Data were not collected on type of cancer therapy. At each interview, use of estrogens (oral contraceptives and/or estrogen replacement therapy (ERT)) was assessed, and the women were asked to bring their over-the-counter and prescription medications to the interview for verification. Estrogen use was modeled as a time-varying covariate in repeated measures regressions to account for the potential to change or stop medication.

The women were asked if any maternal or paternal first-degree relative had been diagnosed as having osteoporosis, or had dowager's hump or "weak bones" (a term used to describe osteoporosis among health care providers around the community). Family history of osteoporosis was defined as any positive report through $1998 / 19999$ and was modeled as a summary variable. Personal history of any fracture was assessed by self-report and modeled as a summary variable.

\section{Statistical analysis}

Univariate statistics were computed for each continuous variable, and frequencies were calculated for categorical variables. BMD, reported as t scores, was normally distributed. Dietary variables with highly skewed distributions were transformed by use of a logarithmic function or were categorized. Independent variables were preliminarily tested for independent associations with BMD $\mathrm{t}$ score via multiple linear regression. We then tested the same independent variables for associations with bone loss, using longitudinal linear mixed models that included time, the independent variable, and their interaction, to assess whether the rate of bone loss is modified by the independent variable. In final analyses, variables that demonstrated either a cross-sectional association with BMD $\mathrm{t}$ score or an association with BMD change were incorporated into linear mixed models as main effects and/or as interactions with time, via linear mixed models (PROC MIXED in SAS) [31]. These models use all available data for each subject, regardless of whether the subjects represent complete cases (having all five BMD measurements). All subjects with at least one BMD measurement contribute to the estimates of association. In addition, these models account for the within-person correlation among the repeated measurements for a given subject [32].

The linear mixed models were of the form

$B M D_{i j}=\mathrm{B}_{o}+\mathrm{B}_{1} t_{i m e} e_{i j}+\mathrm{B}_{2} x_{i j}+\mathrm{B}_{3} x_{i j} \times t i m e_{i j}+b_{i}+\varepsilon_{i j}$

where $\mathrm{BMD}_{\mathrm{ij}}$ is the value of the BMD $\mathrm{t}$ score for the $\mathrm{i}^{\text {th }}$ person at time $\mathrm{j}$, time $\mathrm{ij}_{\mathrm{ij}}$ is the $\mathrm{j}^{\text {th }}$ time point for subject $\mathrm{i}, \mathrm{x}_{\mathrm{ij}}$ is the value of risk factor, $x$, for the $i^{\text {th }}$ person at time $j, b_{i}$ is a random intercept term to account for correlated errors among repeated measures on the same woman, and $\epsilon_{\mathrm{ij}}$ is an error term. The $\mathrm{B}_{1}$ (time) coefficient provides an estimate of the average annual rate of bone loss for premenopausal (the referent group of) women. The $\mathrm{B}_{2}$ (risk factor) coefficient provides an estimate for the difference in BMD t score associated with a unit change in the given risk factor. A significant $\mathrm{B}_{2}$ (risk factor) coefficient is interpreted as a difference in baseline BMD according to level of the risk factor only if there is a corresponding time* risk factor interaction. Otherwise, the $\mathrm{B}_{2}$ (risk factor) coefficient can be interpreted as the association between the risk factor and BMD t score at any time over the 6-year study. The $\mathrm{B}_{3}$ (time by risk factor interaction) coefficient estimates the association between the same risk factor and rate of bone loss. Referent rates of bone loss are those for premenopausal women who have referent values for all other risk factors in the model. Negative beta coefficients for the interaction terms imply additional BMD loss relative to these referent rates, and positive beta coefficients for these interaction terms imply a gain in BMD $\mathrm{t}$ score over time relative to these referent rates (or less BMD loss).

\section{Results}

Baseline characteristics of the study population are presented in Table 1 . Over $70 \%$ of women were aged 35 years and above (age range, 24-44 years). At baseline, $40 \%$ of women were of normal weight, $30 \%$ of women were overweight, and $24 \%$ of women were obese or severely obese. By the 1998/1999 follow-up, the proportion of women who were obese or severely obese had increased to $33 \%$ (data not shown). At baseline, over $90 \%$ of women were premenopausal, and $6 \%(n=37)$ of women were postmenopausal, the majority of whom $(n=34)$ had had a bilateral oophorectomy. By 1998/ $1999,75 \%$ of women remained premenopausal, and another $9 \%$ of women had become postmenopausal (data not shown). The majority of the women on ERT at baseline were those who had had a bilateral oophorectomy. The mean (SD) daily dietary calcium intake was 
Table 1 Baseline characteristics of the study population, Tecumseh, Michigan, 1992/1993 $(n=577)$

\begin{tabular}{lll}
\hline Characteristic & $n$ & $\%$ \\
\hline Age (years) & & \\
24-29 & 46 & 8.0 \\
30-34 & 125 & 21.7 \\
35-039 & 203 & 35.2 \\
40-44 & 203 & 35.2 \\
Body mass index (kg/m ${ }^{2}$ ) & & \\
<20 (underweight) & 36 & 6.2 \\
20-24.9 (normal weight) & 230 & 39.9 \\
25-29.9 (overweight) & 172 & 29.8 \\
30-39.9 (obese) & 117 & 20.3 \\
>40 (severely obese) & 22 & 3.8 \\
Menopausal status & & \\
Premenopausal & 522 & 90.5 \\
Irregular menses & 18 & 3.1 \\
Postmenopausal & 37 & 6.4 \\
Use of estrogens in past year & & \\
Yes & 38 & 6.6 \\
No & 539 & 93.4 \\
Daily dietary calcium intake (mg) [x, (SD)] & 758 & $(455)$ \\
BMD t score & & \\
Spine [x, (SD)] & 0.77 & $(1.25)$ \\
Femoral neck [x, (SD)] & 0.12 & $(1.17)$ \\
\hline
\end{tabular}

${ }^{\mathrm{a}} n=576$ for baseline BMD of the lumbar spine and $n=574$ for baseline BMD of the femoral neck

758 (455) $\mathrm{mg}$ (median $667 \mathrm{mg}$ ). The mean (SD) baseline BMD t scores were $0.77(1.25)$ and $0.12(1.17)$ at the lumbar spine and the femoral neck, respectively. As shown in Table $2,43 \%$ of women reported having participated in high-school sports. At baseline, almost $80 \%$ of women reported consuming modest amounts of alcohol in the past year, and $58 \%$ of women reported never smoking cigarettes. Cancer of the breast, ovary or uterus was reported by $2 \%$ of women. A family history of osteoporosis was reported by $36 \%$ of women, and $41 \%$ of women reported a positive history of any personal fracture.

Linear mixed regression models were developed from the repeated BMD measures to identify risk factors associated with the 6-year rate of BMD change while simultaneously assessing the effects of risk factors on BMD $t$ score at baseline and over the 6-year study period (Table 3). Thus, body weight was associated with both greater BMD t score at baseline and with a slower rate of BMD loss. Specifically, for each additional kilogram of body weight, premenopausal women had, on average, BMD t scores that were 0.01 greater (SE $0.001, P<0.0001$ ), at both skeletal sites and rates of BMD loss that were attenuated by $0.08 \%$ of a t score per year (SE 0.0001, $P<0.0001$ ) at the lumbar spine and by $0.06 \%$ of a t score per year (SE $0.0001, P<0.0001$ ) at the femoral neck.

Women who reported a family history of osteoporosis had BMD t scores that were 0.23 lower at the femoral neck (SE 0.09, $P<0.002$ ), but at the lumbar spine, the association was of borderline significance $(P=0.06)$, and they had no significantly increased rate of BMD loss. Women who reported a history of fracture
Table 2 Frequency distributions of behavioral and medical risk factors among the study population, Tecumseh, Michigan $(n=614)$

\begin{tabular}{|c|c|c|}
\hline Factor & $n$ & $\%$ \\
\hline \multicolumn{3}{|l|}{ Lifestyle } \\
\hline \multicolumn{3}{|c|}{ Participated in high school sports ${ }^{\mathrm{a}}$} \\
\hline Yes & 241 & 42.9 \\
\hline No & 321 & 57.1 \\
\hline \multicolumn{3}{|c|}{ Drank alcohol in past year ${ }^{b}$} \\
\hline Yes & 451 & 78.4 \\
\hline \multirow{2}{*}{\multicolumn{3}{|c|}{ Lifetime smoking history $^{\mathrm{b}}$}} \\
\hline & & \\
\hline Never smoker & 332 & 57.9 \\
\hline Ex-smoker & 115 & 20. \\
\hline Current smoker & 126 & 22.0 \\
\hline \multicolumn{3}{|l|}{ Reproductive history } \\
\hline \multicolumn{3}{|c|}{ Age at menarche (years) ${ }^{\mathrm{d}}$} \\
\hline$\leq 10$ & 33 & 5.5 \\
\hline $11-14$ & 523 & 86.7 \\
\hline$\geq 15$ & 47 & 7.8 \\
\hline \multicolumn{3}{|c|}{ Number of pregnancies ${ }^{b}$} \\
\hline 0 & 103 & 18.0 \\
\hline 1 & 73 & 12.7 \\
\hline 2 & 216 & 37.7 \\
\hline 3 & 132 & 23.0 \\
\hline$\geq 4$ & 50 & 8.7 \\
\hline \multicolumn{3}{|c|}{ Breastfeeding history ${ }^{c}$} \\
\hline Ever & 325 & 36.0 \\
\hline Never & 183 & 64.0 \\
\hline \multicolumn{3}{|l|}{ Medical history ${ }^{\mathrm{d}}$} \\
\hline \multicolumn{3}{|c|}{ Cancer (of breast, ovary or uterus) } \\
\hline Yes & 15 & 2.4 \\
\hline No & 599 & 97.6 \\
\hline \multicolumn{3}{|c|}{ Family history of osteoporosis } \\
\hline Yes & 219 & 36.1 \\
\hline No & 388 & 63.9 \\
\hline \multicolumn{3}{|c|}{ Personal fracture history } \\
\hline Yes & 237 & 40.6 \\
\hline No & 347 & 59. \\
\hline
\end{tabular}

${ }^{\text {a }}$ Measured in 1993/1994 of 567 participants

${ }^{\mathrm{b}}$ Measured in 1992/1993 of 580 participants

${ }^{\mathrm{c}}$ Measured in 1995/1996 of 508 participants

${ }^{\mathrm{d}}$ Measured from all available data through 1998-1999

had BMD $t$ scores at the lumbar spine that were 0.29 lower (SE 0.10, $P=0.005$ ) than women with no reported fracture history, but no significantly added rate of BMD loss. Women who reported alcohol consumption had slightly (0.08) greater BMD t scores (SE 0.02, $P=0.0002$ ) at the femoral neck but no significantly different rate of BMD change than women who reported abstinence. Women who reported high-school sports participation had BMD t scores that were much (0.28) greater (SE 0.09, $P=0.002$ ) at the femoral neck but no significantly different rate of BMD change than women who did not.

The baseline BMD $t$ scores of the lumbar spine for postmenopausal women were similar to those of premenopausal women, but postmenopausal women experienced an additional loss of $4 \%$ of a BMD t score per year (SE 0.01, $P<0.0001$ ) relative to premenopausal women. The baseline BMD $t$ scores of the femoral neck for postmenopausal women were 0.11 lower (SE 0.06, $P=0.04)$ and rates of BMD change were $2 \%$ of a BMD t score per year greater than the rate experienced by 
Table 3 Multiple linear mixed model regression of BMD t score from 1992/1993-1998/1999 by skeletal site, Tecumseh, Michigan

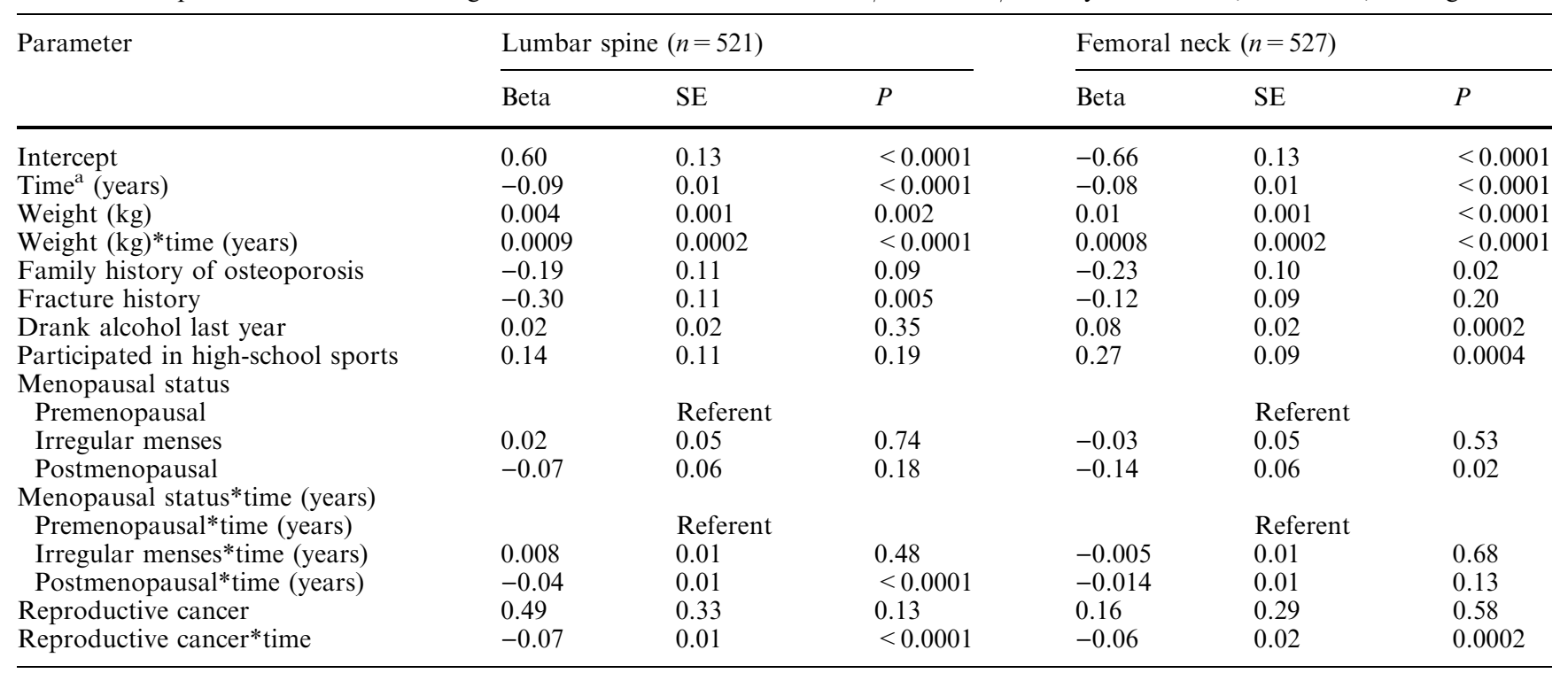

${ }^{\text {a }}$ Time represents annual rate of BMD change for women with regular menstrual cycles and referent values for each risk factor

premenopausal women (SE 0.01, $P=0.01$ ). Women who reported cancer (of the breast, ovary, or uterus) had no different BMD $t$ score at baseline, but lost an additional $7 \%$ of a BMD t score annually (SE $0.01, P<0.0001$ ) at the lumbar spine and $5 \%$ of a BMD t score annually (SE $0.01, P=0.0008)$ at the femoral neck, relative to premenopausal women with no report of cancer, although these inferences are based on few reported cancers.

Notably, daily intakes of calcium, vitamin D, vitamin A and vitamin $\mathrm{C}$ showed no linear associations with BMD t score at either skeletal site. Low dietary intake of calcium (defined as $\leq 400 \mathrm{mg}$ /day) had no association with BMD $t$ score either alone or when tested as an interaction with low dietary vitamin $\mathrm{D}$ ( $\leq 40 \mathrm{IU} /$ day), current physical activity, or past physical activity (data not shown). Supplemental calcium use (yes/no) demonstrated no association with BMD t score either when measured alone or when quantified and added to dietary calcium intake. Neither baseline age nor smoking history showed no crude association with BMD t score at either skeletal site. Neither number of pregnancies nor breastfeeding history demonstrated any relationship with BMD $\mathrm{t}$ score at either skeletal site after we had adjusted for the effects of menopausal status and body weight.

\section{Discussion}

Ours is the first study to simultaneously examine a large set of potential risk factors for lower premenopausal BMD and to disaggregate the effects of these risk factors on BMD status from their effects on BMD loss. Risk factors for lower BMD among premenopausal women included lower body weight, postmenopausal status, history of fracture, family history of osteoporosis, and non-participation in high-school sports. Risk factors for greater rate of bone loss included postmenopausal status and a reported reproductive cancer. Dietary calcium consumption, current physical activity, number of pregnancies, breastfeeding, and smoking were not associated with either BMD level or rate of BMD change in this well-nourished and relatively sedentary population.

Body weight was positively associated with BMD of the lumbar spine and femoral neck among premenopausal women [33, 34, 35]. Greater body weight also protected against the slight bone loss that occurs naturally prior to menopause [2]. The mitigating effect of weight on bone loss has been previously demonstrated among postmenopausal $[36,37,38]$ and perimenopausal [39] women but not, to our knowledge, in premenopausal women. Body weight might affect BMD through any of several hypothesized mechanisms. A larger body mass imposes a greater mechanical strain on bone, and in response, bone mass may increase to accommodate the greater load [40]. Alternatively, increased peripheral conversion of androstenedione to estrone in the larger adipose tissue mass among heavier, usually postmenopausal, women might result in increased inhibition of enzymatic bone matrix degradation by osteoclasts [41]. Other hormonal mechanisms might include increased circulating insulin, amylin and/or leptin and their potential regulating effect on bone tissue, via increased ovarian estrogen production or decreased levels of sex hormone binding globulins resulting in either osteoblast proliferation or osteoclast inhibition [42]. Because body weight explains a greater proportion of the variance in BMD of the femoral neck (a weight-bearing site) than the lumbar spine (18\% vs $8.5 \%)$, these data suggest that mechanical loading is the predominant mechanism among premenopausal women. If the effect of weight 
were mainly of a hormonal nature, the cancellous bone of the lumbar spine should have been influenced at least as strongly as the femoral neck.

Greater rates of bone loss occurred among the few women who had reported a diagnosis of a reproductive (breast, ovarian, or uterine) cancer. In contrast to studies in older women, where BMD has been viewed as a marker of cumulative exposure to estrogens and, therefore, as a potential marker for increased risk of postmenopausal breast cancer [43, 44], the effect we describe may be related to premature ovarian failure secondary to chemotherapy treatment [19] for premenopausal cancers. The higher rates of bone loss experienced by the postmenopausal women were, by and large, an effect of bilateral oophorectomy. In separate analyses, women with bilateral oophorectomy had substantially greater bone loss at the femoral neck than women with natural menopause, despite frequent reports of ERT use. These findings underscore the importance of iatrogenic bone loss secondary to cancer and bilateral oophorectomy and certainly would provide incentive for the clinician to consider a bone health assessment for women experiencing either cancer or surgical menopause.

Women who reported a family history of osteoporosis had a lower BMD than women who reported no family history. Numerous investigations have suggested that, among daughters of women with osteoporosis, a stronger association with low BMD exists for premenopausal daughters than postmenopausal daughters [45]. In our study, recalled family history was associated with BMD level but not with rate of bone loss. Future study will be required to discern if the familial component of BMD is also related to age at menopause or rate of bone loss in the immediate postmenopause.

There was an association between history of fracture and lower BMD of the lumbar spine, a result consistent with several other studies [34, 46, 47, 48]. No similar association of fracture history was found for BMD of the femoral neck or for rate of bone loss at either skeletal site. Previous fracture may be acting as a marker for familial or environmental conditions predisposing to low BMD. Alternatively, if the reported fractures were in the recent past, history of fracture might be a marker for bone loss due to disuse osteodystrophy following fracture.

Among all the lifestyle risk factors examined, highschool sports participation appeared to have the greatest influence on BMD level, although the effect was limited to the femoral neck and did not affect rate of BMD change. The importance of past physical activity for premenopausal BMD supports evidence that adolescence is a critical period for bone mass accrual [49], during which time physical activity may play a crucial part [50]. In this study, high-school sports participation may be measuring another correlate of BMD, such as adolescent lean mass or a social or other behavioral factor. Whether or not physical activity of all types and intensities affects BMD accrual remains uncertain. No effect of current physical activity was detected. This null finding may be due to our inability to separate loadbearing activities from those that are non-load-bearing.

Women who reported modest alcohol consumption had slightly higher femoral neck BMD than women who abstained. Other investigators have also reported that modest alcohol consumption is positively associated with BMD [51, 52], an effect that contrasts the recognized impact of alcoholism on bone [53]. While the mechanism for such an association is currently unknown, hormonal effects, such as greater central adiposity and increased serum estradiol [54], increased calcitonin secretion [55], or hypoparathyroidism [56], are possible. Modest alcohol consumption did not affect rates of bone change in our study, and the findings should not be extended to justify increasing one's alcohol consumption.

The belief that smoking is a major risk factor for osteoporosis has propagated since the publication of a study conducted among thin, estrogen-deficient, postmenopausal women [57] Smoking is hypothesized to affect premenopausal BMD by altering the sex steroid hormonal environment [58, 59], though the effect can also be attributed to a lower average body weight among smokers [60]. Consistent with a recent meta-analysis of smoking and BMD [16], no effect of smoking on BMD or bone loss was identified in our population of somewhat heavy, mainly premenopausal, women. Potentially, smoking may be measurably deleterious to bone health only among thin, estrogen-deficient women.

No association was detected between dietary calcium and BMD or BMD loss, even among sedentary women or those with low dietary vitamin D intake. Our study adds to increasing evidence that BMD or bone change in young or middle-aged women is related to factors other than overt calcium deficiency [8]. The promotion of calcium consumption as the panacea of bone mineral maintenance among healthy premenopausal and perimenopausal women may be inappropriate.

This longitudinal study had enough power to detect a large number of independent risk factors for both low BMD and for bone loss among a population of women approaching the menopausal transition. Melton et al. have recently demonstrated that factors related to bone loss are different for premenopausal women than for postmenopausal women who are within 20 years of menopause and different still for women whose menopause occurred over 20 years ago [3]. Therefore, the risk factors for bone loss among premenopausal women identified in this study must be considered carefully in relation to bone loss among postmenopausal women or to the risk of fracture.

The longitudinal methods employed in this study included the ability to reclassify an exposure that may change with time (such as alcohol consumption) and to accumulate events (such as fractures) over time. These methods may have diminished any misclassification bias and increased the power necessary to detect associations with BMD. Using these longitudinal methods, this study was able to discern that certain risk factors are related to 
BMD at any given point in time, but not to rate of bone loss.

Study limitations include a number of measures based on participant recall. While precise measurements of physical activity [61] and food intake [62] are notoriously difficult, repeated measurements should have decreased the amount of random measurement error. This Caucasian study population did not allow the examination of risk factors among women of other racial and ethnic backgrounds. Because this population is just beginning to reflect the postmenopausal experience, it is not yet possible for one to determine the relative effects of these risk factors on BMD through to the postmenopause. Although this study is able to assess temporal relationships between a variety of risk factors and BMD and BMD loss, the study design is observational and unable to assign causality without corroborative evidence.

This longitudinal study provides new understanding of which risk factors appear to be antecedent determinants of current BMD (and possibly correlated with peak bone mass) and those that influence bone loss as women approach and enter the postmenopausal period. Our understanding of this distinction should enhance the effectiveness of osteoporosis prevention efforts by matching interventions to women of the appropriate life stage. The women at greatest risk for postmenopausal osteoporosis may be those who exhibit one or more traits from both groups of risk factors: those related to BMD status and those related to BMD loss. Finally, the recognition of these risk factors in younger populations should promote earlier introduction of appropriate intervention.

Acknowledgement Funding was obtained by AR R01-40888 (Sowers, PI) and AR P60-20557 (Sowers, PI). We acknowledge Mary Crutchfield and Mary Jannausch for their contributions to data collection and management.

\section{References}

1. Sowers MF (2000) Lower peak bone mass and its decline. Baillieres Clin Endocrinol Metab 14:317-329

2. Bainbridge KE, Sowers MF, Crutchfield M, et al (2002) Natural history of bone loss over 6 years among premenopausal and early postmenopausal women. Am J Epidemiol 156:410417

3. Melton LJ, Atkinson EJ, O'Connor MK, et al (2000) Determinants of bone loss from the femoral neck in women of different ages. J Bone Miner Res 15:24-31

4. Sowers MF, Crutchfield M, Bandekar R, et al (1998) Bone mineral density and its change in pre- and perimenopausal white women: The Michigan Bone Health Study. J Bone Miner Res 13:1124-1140

5. Revilla M, Villa LF, Hernandez ER, et al (1997) Influence of weight and gonadal status on total and regional bone mineral content and on weight-bearing and non-weight bearing bones, measured by dual-energy X-ray absorptiometry. Maturitas 28:69-74

6. Sowers MF, Kshirsagar A, Crutchfield MM, et al (1992) Joint influence of fat and lean body composition compartments on femoral bone mineral density in premenopausal women. Am J Epidemiol 136:257-265
7. Snow-Harter CM (1994) Bone health and prevention of osteoporosis in active and athletic women. Clin Sports Med 13:389404

8. Sowers MFR, Galuska DA (1993) Epidemiology of bone mass in premenopausal women. Epidemiol Rev 15:374-398

9. Sowers MF (1996) Pregnancy and lactation as risk factors for subsequent bone loss and osteoporosis. J Bone Miner Res 11:1052-1060

10. Lloyd T, Taylor DS (2001) Calcium intake and peak bone mass. J Am Med Womens Assoc 56:49-52

11. Tudor-Locke C, McColl RS (2000) Factors related to variation in premenopausal bone mineral status: a health promotion approach. Osteoporos Int 11:1-24

12. Hall SL, Greendale GA (1998) The relation of dietary vitamin $\mathrm{C}$ intake to bone mineral density: results from the PEPI study. Calcif Tissue Int 63:183-189

13. Leveille SG, LaCroix AZ, Koepsell TD, et al (1997) Dietary vitamin $\mathrm{C}$ and bone mineral density in postmenopausal women in Washington State, USA. J Epidemiol Community Health $51: 479-485$

14. Sowers MFR, Wallace RB (1990) Retinol, supplemental vitamin A and bone status. J Clin Epidemiol 43:693-699

15. Laitinen K, Valimaki M (1991) Alcohol and bone. Calcif Tissue Int 49[Suppl]:S70-S73

16. Law MR, Hackshaw AK (1997) A meta-analysis of cigarette smoking, bone mineral density and risk of hip fracture: recognition of a major effect. BMJ 315:841-846

17. Snow CM (1996) Exercise and bone mass in young and premenopausal women. Bone 18:51S-55S

18. Laatikainen AK, Kroger HP, Tukiainen HO, et al (1999) Bone mineral density in perimenopausal women with asthma: a population-based cross-sectional study. Am J Respir Crit Care Med 159:1179-1185

19. Pfeilschifter J, Diel IJ (2000) Osteoporosis due to cancer treatment: pathogenesis and management. J Clin Oncol 18:1570-1593

20. Pollitzer WS, Anderson JJB (1989) Ethnic and genetic differences in bone mass: a review with a hereditary vs environmental perspective. Am J Clin Nutr 50:1244-1259

21. Hunter DJ, de Lange M, Andrew T, et al (2001) Genetic variation in bone mineral density and calcaneal ultrasound: a study of the influence of menopause using female twins. Osteoporos Int 12:406-411

22. Lips P (1997) Epidemiology and predictors of fractures associated with osteoporosis. Am J Med 103:20S-26S

23. Gilfillan C, Silberberg S, Scrivenor P, et al (1994) Determinants of forearm mineral density and its correlation with fracture history in women. Maturitas 20:199-208

24. Honkanen R, Kroger H, Tuppurainen M, et al (1995) Fractures and low axial bone density in perimenopausal women. J Clin Epidemiol 48:881-888

25. Mazess RB, Barden HS (1991) Bone density in premenopausal women: effects of age, dietary intake, physical activity, smoking, and birth control pills. Am J Clin Nutr 53:132-142

26. Bray GA (1987) Overweight is risking fate: definition, classification, prevalence and risks. Ann N Y Acad Sci 499:14 28

27. Block G, Hartman AM, Dresser CM, et al (1986) A data-based approach to diet questionnaire design and testing. Am J Epidemiol 124:453-469

28. National Cancer Institute (1993) HHHQ-DIETSYS Analysis software, version 3.0

29. Bowes and Church's food values of portions commonly used (1980) In: Pennington JAT, Church HN (eds). Harper and Row, New York, NY, USA

30. Sallis JF, Haskell WL, Wood PD, et al (1985) Physical activity assessment methodology in the five-city project. Am J Epidemiol 121:91-106

31. SAS proprietary software, release 6.12; SAS Institute, Cary, NC, USA

32. Diggle PJ, Liang K-Y, Zeger S (1994) Analysis of longitudinal data. Clarendon Press, Oxford 
33. Sowers MF, Kshirsagar A, Crutchfield M, et al (1991) Body composition, age and femoral bone mass of young adult women. Ann Epidemiol 1:245-254

34. Torgerson DJ, Campbell MK, Reid DM (1995) Life-style, environmental and medical factors influencing peak bone mass in women. Br J Rheumatol 34:620-624

35. New SA, Bolton-Smith C, Grubb DA, et al (1997) Nutritional influences on bone mineral density: a cross-sectional study in premenopausal women. Am J Clin Nutr 65:1831-1839

36. Harris S, Dallal GE, Dawson-Hughes B (1992) Influence of body weight on rates of change in bone density of the spine, hip, and radius in postmenopausal women. Calcif Tissue Int 50:19-23

37. Tremollieres FA, Pouilles J-M, Ribot C (1993) Vertebral postmenopausal bone loss is reduced in overweight women: a longitudinal study in 155 early postmenopausal women. J Clin Endocrinol Metab 77:683-696

38. Ravn P, Cizza G, Bjarnason NH, et al (1999) Low body mass index is an important risk factor for low bone mass and increased bone loss in early postmenopausal women. J Bone Miner Res 14:1622-1627

39. Brot C, Jensen LB, Sorensen OH (1997) Bone mass and risk factors for bone loss in perimenopausal Danish women. J Int Med 242:505-511

40. Rubin CT, Lanyon LE (1985) Regulation of bone mass by mechanical strain magnitude. Calcif Tissue Int 37:411-417

41. Reid IR, Plank LD, Evans MC (1992) Fat mass is an important determinant of whole body bone density in premenopausal women but not in men. J Clin Endocrinol Metab 75:779-782

42. Reid IR (2002) Relationships among body mass, its components, and bone. Bone 31:547-555

43. Zmuda JM, Cauley JA, Ljung B-M, Bauer DC, Cummings SR, Kuller LH (2001) Bone mass and breast cancer risk in older women: differences by stage at diagnosis. J Natl Cancer Inst 93:930-936

44. Zhang Y, Kiel DP, Kreger BE, Cupples LA, Ellison C, Dorgan JF, Schatzkin, A, Levy D, Felson DT (1997) Bone mass and the risk of breast cancer among postmenopausal women. N Engl J Med 336:611-617

45. Danielson ME, Cauley JA, Baker CE, et al (1999) Familial resemblance of bone mineral density (BMD) and calcaneal ultrasound attenuation: the BMD in mothers and daughters study. J Bone Miner Res 14:102-110

46. Cox ML, Khan SA, Gau DW, et al (1991) Determinants of forearm bone density in premenopausal women: a study in one general practice. Br J Gen Pract 41:194-196

47. Goulding A, Gold E, Walker R, et al (1997) Women with past history of bone fracture have low spinal bone density before menopause. N Z Med J 110:232-233
48. Honkanen R, Kroger H, Tuppurainen M, et al (1995) Fractures and low axial bone density in perimenopausal women. J Clin Epidemiol 48:881-888

49. Sabatier JP, Guaydier-Souquieres G, Benmalek A, et al (1999) Evolution of lumbar bone mineral content during adolescence and adulthood: a longitudinal study in 395 healthy females 10 24 years of age and 206 premenopausal women. Osteoporos Int 9:476-482

50. Lloyd T, Chinchilli VM, Johnson-Rollings N, et al (2000) Adult female hip bone density reflects teenage sports-exercise patterns but not teenage calcium intake. Pediatrics 106:40-44

51. Feskanich D, Korrick SA, Greenspan SL, et al (1999) Moderate alcohol consumption and bone density among postmenopausal women. J Womens Health 8:65-73

52. Holbrook TL, Barrett-Connor E (1993) A prospective study of alcohol consumption and bone mineral density. BMJ 306:15061509

53. Clark K, Sowers MF (1996) Alcohol dependence, smoking status, reproductive characteristics, and bone mineral density in premenopausal women. Res Nurs Health 19:399-408

54. Reichman ME, Judd JT, Longcope C, et al (1993) Effects of alcohol consumption on plasma and urinary hormone concentrations in premenopausal women. J Natl Cancer Inst $85: 722-727$

55. Rico H (1990) Alcohol and bone disease. Alcohol Alcohol $25: 345-352$

56. Laitinen K, Lamberg-Allardt C, Tunninen R, et al (1991) Transient hypoparathyroidism during acute alcohol intoxication. N Engl J Med 324:721-727

57. Daniell HW (1976) Osteoporosis of the slender smoker. Vertebral compression fractures and loss of metacarpal cortex in relation to postmenopausal cigarette smoking and lack of obesity. Arch Intern Med 136:298-304

58. Ortego-Centeno N, Munoz-Torres M, Hernandez-Quero J, et al (1994) Bone mineral density, sex steroids, and mineral metabolism in premenopausal smokers. Calcif Tissue Int 55:403-407

59. Michnovicz JJ, Hershcopf RJ, Naganuma H, et al (1986) Increased 2-hydroxylation of estradiol as a possible mechanism for the anti-estrogenic effect of cigarette smoking. N Engl J Med 315:1305-1309

60. Jensen GF (1986) Osteoporosis of the slender smoker revisited by epidemiologic approach. Eur J Clin Invest 16:239-242

61. LaPorte RE, Montoye HJ, Casperson CJ (1985) Assessment of physical activity in epidemiologic research: problems and prospects. Public Health Rep 100:131-146

62. Todd KS, Hudes M, Calloway DH (1983) Food intake measurement: problems and approaches. Am J Clin Nutr 37:139146 